\title{
The Natural History of Facial Schwannomas: A Meta-Analysis of Case Series
}

\author{
Matthew Bartindale ${ }^{1}$ Jeffrey Heiferman ${ }^{1}$ Cara Joyce ${ }^{2}$ Neelam Balasubramanian ${ }^{2}$ \\ Douglas Anderson ${ }^{3}$ John Leonetti ${ }^{1}$
}

${ }^{1}$ Depaent of Otolaryngology-Head and Neck Surgery, Loyola University Medical Center, Maywood, Illinois

${ }^{2}$ Clinical Research Office, Division of Biostatistics, Loyola University

Medical Center, Maywood, Illinois

${ }^{3}$ Department of Neurological Surgery, Loyola University Medical

Center, Maywood, Illinois

J Neurol Surg B 2019;80:458-468.
Address for correspondence John Leonetti, MD, Department of Otolaryngology-Head and Neck Surgery, Loyola University Medical Center, Maguire Building, 2160 South. First Avenue, Maywood, IL 60153 United States (e-mail: jleonet@lumc.edu).

\begin{abstract}
Keywords

- facial schwannoma

- facial nerve

- facial palsy

- hearing loss

- parotid neoplasm

- temporal bone neoplasm

- cerebellopontine angle neoplasm

Objective This study is to establish predictors of facial paralysis and auditory morbidity secondary to facial schwannomas by assimilating individualized patient data from the literature.

Design A systematic review of the literature was conducted for studies regarding facial schwannomas. Studies were only included if they presented patient level data, House-Brackmann grades, and tumor location by facial nerve segment. Odds ratios (OR) were estimated using generalized linear mixed models.

Main Outcome Measures Facial weakness and hearing loss.

Results Data from 504 patients were collected from 32 studies. The geniculate ganglion was the most common facial nerve segment involved (39.3\%). A greater number of facial nerve segments involved was positively associated with both facial weakness and hearing loss, whereas tumor diameter did not correlate with either morbidity. Intratemporal involvement was associated with higher odds of facial weakness $(\mathrm{OR}=4.78, p<0.001)$, intradural involvement was negatively associated with facial weakness ( $O R=0.56, p=0.004)$, and extratemporal involvement was not a predictor of facial weakness $(O R=0.68, p=0.27)$. The odds of hearing loss increased with more proximal location of the tumor (intradural: $O R=3.26$, $p<0.001$; intratemporal: $\mathrm{OR}=0.60, p=0.14$; extratemporal: $\mathrm{OR}=0.27, p=0.01$ ). Conclusion The most important factors associated with facial weakness and hearing loss are tumor location and the number of facial nerve segments involved. An understanding of the factors that contribute most heavily to the natural morbidity can help guide the appropriate timing and type of intervention in future cases of facial schwannoma.
\end{abstract}

\section{Introduction}

Facial schwannomas are benign tumors originating from the myelin producing schwann cell sheath. ${ }^{1}$ These tumors are so rare that determining their true incidence has been difficult; however, it is thought to be the most common primary neo-

received

February 10, 2018

accepted after revision

September 23, 2018

published online

November 21, 2018

plasm of the facial nerve. ${ }^{2}$ Schwannomas can be found anywhere along the facial nerve and skip lesions have been described. ${ }^{3}$ It can be difficult to distinguish a facial schwannoma from a vestibular schwannoma based on imaging alone, particularly when it involves only the internal auditory canal (IAC) or cerebellopontine angle (CPA). The distinction is primarily made

(c) 2019 Georg Thieme Verlag KG Stuttgart · New York
DOI https://doi.org/ 10.1055/s-0038-1675590. ISSN 2193-6331. 
based on clinical symptoms. ${ }^{4}$ The clinical presentation of facial schwannoma is variable but usually consists of facial weakness, hearing loss, and/or a parotid mass. ${ }^{2}$

Facial schwannomas create a management dilemma because the morbidity of the treatment can be greater than the morbidity of the natural course of disease. Considerable controversy surrounds the treatment of these tumors. Fortunately, most facial schwannomas are slow growing, and many can be observed for years. ${ }^{5}$ Growth rates have been reported between $0.4^{6}$ and $2.0 \mathrm{~mm} /$ year. $^{7}$ This creates a delicate balance when considering the timing and type of treatment. The primary goal of treatment is to preserve facial nerve function for the longest duration possible, unless there are other factors making intervention absolutely necessary. ${ }^{2}$

Historically, the treatment options for facial schwannomas were observation with radiographic surveillance, facial schwannoma decompression, and total resection with nerve grafting. Decompression gives the tumor additional space to grow before the facial nerve becomes compressed. However, it is not a definitive treatment and a resection may eventually be required. Even following grafting, the best possible outcome with total resection is House-Brackmann (HB) grade III. $^{2}$ Most facial schwannomas were observed until facial nerve function deteriorated to $\mathrm{HB}$ grade IV or worse at which point intervention was considered. More recently, new treatment options and consideration of a new treatment paradigm have emerged. Subtotal resection, or nerve "stripping" surgery, with tumor debulking can be attempted to spare the facial nerve. ${ }^{1,8-10}$ Radiation therapy, as with vestibular schwannomas, has also emerged as a treatment option in certain scenarios that avoids operative intervention. ${ }^{11-13}$ Regardless of the type of treatment, the timing of the treatment is also controversial. The management discussion varies depending on the location of the tumor due to the variable morbidities of the approaches required.

Several factors make the research to determine a definitive treatment algorithm challenging. First, the rarity of the neoplasm limits the sample size in published case series. This rarity is exacerbated because of the wide variability in anatomic locations of facial schwannomas. For example, intraparotid facial schwannomas are managed completely differently than intradural facial schwannomas. The variability in clinical presentation also plays a part, particularly with regards to facial nerve function and hearing status. A patient with HB grade I will have different treatment options than a patient with HB grade VI.

Our objective in this study is to establish predictors of morbidity secondary to facial schwannomas.

\section{Methods}

This study was performed according to PRISMA (Preferred Reporting Items for Systematic Reviews and Meta-analysis) guidelines. ${ }^{14}$ The review protocol was registered on PROSPERO (International Prospective Register of Systematic Review) (CRD42016050204). An Institutional Review Board exemption was granted because human subjects were not involved in this study.

\section{Eligibility Criteria}

(1) The reference must have data on individual patients, not aggregated data; (2) each patient must have a preintervention HB grade reported; (3) each patient must have the schwannoma location reported by specific facial nerve segment(s) involved; (4) patients must not have had any prior interventions; (5) patients must not have neurofibromatosis type II; (6) the reference must have at least five eligible patients. References were still included if only a portion of patients were eligible; $(7)$ the reference must contain primary data; (8) the same patient must not have been reported multiple times; (9) the reference must be in English.

\section{Search Strategy}

To identify relevant studies, searches were performed in PubMed-NCBI (National Center for Biotechnology Information) and Scopus by an academic librarian. The search strategies employed are included in the -Appendix A. Only articles in English were included, and only papers after 1985 were used, as that is when the HB grading system was introduced. ${ }^{15}$

\section{Study Selection and Validation}

Two reviewers independently screened each abstract and then evaluated the remaining full articles for eligibility. Discrepancies were resolved by a third reviewer.

\section{Data Abstraction}

Information was extracted at two levels, a study level and a patient level. Information extracted from each study included author, year of publication, number of patients, whether the study was restricted to a specific population based on location or facial nerve status, whether it was retrospective or prospective, and the study's level of evidence based on the Oxford Centre for Evidence Based Medicine 2011 criteria. ${ }^{16}$ Information extracted from individual patients when available, included gender, age, laterality, symptoms at presentation, tumor location by facial nerve segment(s) involved, tumor diameter, tumor volume, preintervention HB grade, and preintervention hearing status. The hearing status was documented as normal or abnormal because of the variability in reporting. Hearing was considered normal if it was American Academy of Otolaryngology-Head and Neck Surgery Class A. ${ }^{17}$ The CPA and IAC segments of the facial nerve were considered intradural; the labyrinthine, geniculate ganglion, tympanic, and mastoid were considered intratemporal; and the parotid segment was considered extratemporal. When more than one paper that met criteria had the same author institution, they were cross referenced to ensure that the same patient was not reported more than once by examining years included and patient details. When a redundancy was seen, the data was omitted from the more recently published study for the applicable patients. The data were entered into an electronic research database (REDCap). ${ }^{18}$

\section{Assessment of Quality and Bias of Individual Studies}

The National Institutes of Health's (NIH) Quality Assessment of Case Series Studies ${ }^{19}$ was used to evaluate quality and bias 
1. Was the study question or objective clearly stated?

2. Was the study population clearly and fully described, including a case definition?

3. Were the cases consecutive?

4. Were the subjects comparable?

5. Was the intervention clearly described?

6. Were the outcome measures clearly defined, valid, reliable, and implemented consistently across all study participants?

7. Was the length of follow-up adequate?

8. Were the statistical methods well-described?

9. Were the results well-described?

Fig. 1 Criteria for the National Institutes of Health's Quality Assessment of case series studies. ${ }^{17}$

of individual studies. - Fig. 1 shows the criteria used in this assessment.

\section{Statistical Methods}

Generalized linear mixed effects models were used to examine associations between patient characteristics and $\mathrm{HB}$ grade accounting for patient clustering using random intercepts for each article. When proportional odds assumptions were met, univariable ordinal logistic mixed effects regression models were specified for each predictor and a cumulative logit link was used to estimate the odds ratios (OR) and 95\% confidence intervals ( $\mathrm{CI}$ ). For those models for which the proportional odds assumption was violated, HB grade was collapsed into 1 to 2 versus 3 to 6 and a binomial distribution was specified for the outcome using a logit link. These HB grade groupings were chosen because of their implications for treatment since the best possible outcome following total resection with grafting is $\mathrm{HB}$ grade III. $^{2}$

Separate generalized linear mixed effects models were used to estimate the odds of preoperative hearing loss as a function of univariable preoperative patient characteristics. As with facial weakness, to account for patient clustering, random intercepts were assigned to each article. A binomial distribution was specified for each model and a logit link was used to estimate the OR and its $95 \% \mathrm{CI}$. All analyses were performed using SAS Version 9.4 (Cary, NC, U.S.A).

\section{Results}

\section{Study Selection}

A total of 605 studies were identified from the PubMed and Scopus searches. After duplicates were removed, 458 abstracts were screened. After implementation of our selection criteria, 396 studies were excluded based on their abstracts. The remaining 62 full articles were reviewed and 32 studies fulfilled all criteria for inclusion. The reasons for exclusion of studies are listed in - Fig. $\mathbf{2}$.

\section{Study Characteristics}

The studies that met inclusion criteria were published between 2000 and 2016. - Table 1 describes the characteristics of each study. A total of 504 patients were included. Preintervention hearing status was described in 18 references and 254 patients. There were 189 patients where the tumor diameter was recorded and 35 patients where the volume was recorded, but none of those studies had multiple preintervention time points to document growth rates. Age was reported for 378 patients, gender in 364 patients, and laterality in 119 patients. Per the inclusion criteria, all patients had documented HB grades and tumor location by facial nerve segment. The assessment of quality and bias for each study was recorded in -Table $\mathbf{2}$.

\section{Epidemiology}

Demographic data, for the patients in which it was recorded, are listed in - Table 3. - Fig. $\mathbf{3}$ shows the age distribution of patients by decade of life. The minimum age is 13 months and the maximum age is 87 years old. -Table 4 shows location characteristics of facial schwannomas by segment and site for all patients included in this study. - Table 5 shows the same location characteristics but it excludes studies that

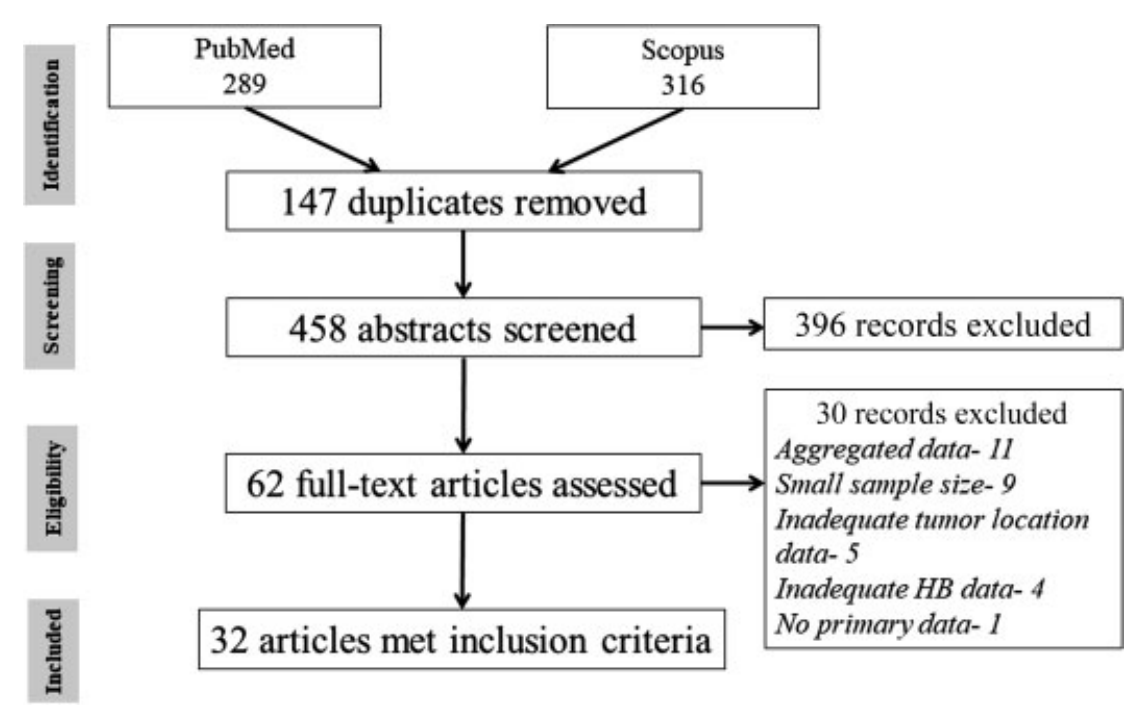

Fig. 2 Study selection process and reasons for exclusion. 
Table 1 Study characteristics

\begin{tabular}{|c|c|c|c|c|c|c|c|c|}
\hline Ref. & First author & PY & $\mathrm{HL}$ & SP & Type of SP & Pts in study & Pts used & Reason for pt removal \\
\hline 20 & Zheng & 2016 & No & Yes & Parotid only & 28 & 28 & \\
\hline 8 & Sun & 2015 & Yes & Yes & Favorable FN function & 18 & 14 & $\begin{array}{l}\text { Redundant patients } \\
\text { from other study }\end{array}$ \\
\hline 21 & Xiang & 2015 & Yes & Yes & Favorable FN function & 19 & 19 & \\
\hline 9 & Lu & 2015 & Yes & Yes & Poor FN function & 17 & 17 & \\
\hline 5 & Yang & 2015 & Yes & Yes & Favorable FN function & 21 & 21 & \\
\hline 22 & Doshi & 2015 & No & No & & 26 & 26 & \\
\hline 11 & Fezeu & 2015 & Yes & No & & 5 & 5 & \\
\hline 12 & Moon & 2014 & Yes & No & & 14 & 9 & Prior treatment \\
\hline 1 & Park & 2014 & Yes & No & & 28 & 28 & \\
\hline 23 & Bacciu & 2014 & Yes & Yes & CPA/IAC only & 23 & 23 & \\
\hline 24 & $\mathrm{Li}$ & 2014 & Yes & No & & 15 & 15 & \\
\hline 25 & Lee & 2013 & No & Yes & Parotid only & 15 & 15 & \\
\hline 26 & Bacciu & 2013 & Yes & Yes & $\begin{array}{l}\text { Complex cases only } \\
\text { with specific criteria }\end{array}$ & 13 & 13 & \\
\hline 27 & $\mathrm{Li}$ & 2012 & No & Yes & Parotid only & 7 & 7 & \\
\hline 28 & Gross & 2012 & No & Yes & Parotid only & 15 & 15 & \\
\hline 29 & Mowry & 2012 & Yes & Yes & CPA/IAC only & 16 & 11 & Inadequate location data \\
\hline 30 & Lee & 2011 & No & No & & 25 & 25 & \\
\hline 31 & Günther & 2010 & Yes & No & & 26 & 26 & \\
\hline 32 & Bäck & 2010 & No & No & Parotid only & 10 & 5 & Inadequate location data \\
\hline 33 & Guzzo & 2009 & No & Yes & Parotid only & 8 & 8 & \\
\hline 34 & McMonagle & 2008 & No & No & & 53 & 52 & Inadequate location data \\
\hline 35 & Kohmura & 2007 & Yes & Yes & CPA/IAC only & 6 & 6 & \\
\hline 36 & Lee JD & 2007 & Yes & Yes & Favorable FN function & 6 & 6 & \\
\hline 37 & Kida & 2007 & Yes & No & & 14 & 6 & $\begin{array}{l}\text { Prior treatment-7, } \\
\text { NF2-1 }\end{array}$ \\
\hline 38 & Litre & 2007 & No & No & & 11 & 9 & Prior treatment \\
\hline 6 & Perez & 2005 & Yes & Yes & Intratemporal only & 24 & 24 & \\
\hline 39 & Minovi & 2004 & No & No & & 11 & 11 & \\
\hline 40 & Chung & 2004 & Yes & No & & 8 & 8 & \\
\hline 10 & Nadeau & 2003 & No & Yes & CPA/IAC only & 7 & 7 & \\
\hline 41 & Kim & 2003 & No & Yes & Intratemporal only & 18 & 18 & \\
\hline 42 & Liu & 2001 & No & No & & 22 & 22 & \\
\hline 43 & Chong & 2000 & Yes & Yes & Parotid only & 5 & 5 & \\
\hline
\end{tabular}

Abbreviations: CPA, cerebellopontine angle; FN, facial nerve; HL, documented hearing loss; IAC, internal auditory canal; NF2, neurofibromatosis type 2; Pts, patients; PY, publication year; Ref., reference number; SP, specific populations.

selected patients from specific populations based on location or facial nerve function. Presenting symptoms were described in 401 patients and their frequencies are listed in - Table 6. The most common presenting symptom for intradural facial schwannoma was hearing loss, for intratemporal tumor was facial weakness, and for extratemporal tumor was a parotid mass. Average tumor diameter was 21.3 $+/-12.0 \mathrm{~mm}(n=189)$ and average tumor volume was $4,167+/-8,387 \mathrm{~mm}^{3}(n=35)$. An effort was made to collect data for tumor growth, but unfortunately there were not enough tumor sizes reported at multiple time points for meaningful results to be reported. The average number of facial nerve segments involved was $2.15+/-1.29$ segments.

\section{Facial Weakness}

- Table 7 shows the analysis of factors associated with facial weakness. Demographics, such as gender, age, and laterality were not predictors of facial weakness. Hearing status also 
Table 2 Assessment of quality and individual bias for individual studies based on the Oxford Centre for Evidence Based Medicine 2011 criteria (OCEBM) ${ }^{16}$ and the Standardized Risk Assessment of Individual Studies based on the NIH Quality Assessment Tool for case series studies. ${ }^{19}$

\begin{tabular}{|c|c|c|c|c|c|c|c|c|c|c|c|c|c|}
\hline Ref. & First author & PY & $\mathrm{P} / \mathrm{R}$ & OCEBM & 1 & 2 & 3 & 4 & 5 & 6 & 7 & 8 & 9 \\
\hline 20 & Zheng & 2016 & $\mathrm{R}$ & 4 & $\mathrm{Y}$ & $Y$ & $\mathrm{Y}$ & $\mathrm{Y}$ & $\mathrm{Y}$ & $\mathrm{Y}$ & $Y$ & $\mathrm{Y}$ & $\mathrm{Y}$ \\
\hline 8 & Sun & 2015 & $R$ & 4 & $\mathrm{Y}$ & $\mathrm{Y}$ & $\mathrm{Y}$ & $\mathrm{Y}$ & $\mathrm{Y}$ & $\mathrm{Y}$ & $Y$ & $\mathrm{NA}$ & $\mathrm{Y}$ \\
\hline 21 & Xiang & 2015 & $R$ & 4 & $Y$ & $\mathrm{Y}$ & $\mathrm{Y}$ & $\mathrm{Y}$ & $\mathrm{Y}$ & $Y$ & $Y$ & $\mathrm{NA}$ & $\bar{Y}$ \\
\hline 8 & $\overline{L u}$ & 2015 & $R$ & 4 & $Y$ & $\mathrm{Y}$ & $\bar{Y}$ & $\bar{Y}$ & $\mathrm{Y}$ & $Y$ & $\mathrm{Y}$ & $\mathrm{NA}$ & $\bar{Y}$ \\
\hline 5 & Yang & 2015 & $R$ & 4 & $\mathrm{Y}$ & $\mathrm{Y}$ & $\mathrm{Y}$ & $\mathrm{Y}$ & $\mathrm{Y}$ & $\mathrm{Y}$ & $Y$ & $\mathrm{NA}$ & $\mathrm{Y}$ \\
\hline 22 & Doshi & 2015 & $R$ & 4 & $\mathrm{Y}$ & $\mathrm{Y}$ & $\mathrm{Y}$ & $\mathrm{Y}$ & $\mathrm{Y}$ & $\mathrm{Y}$ & $\mathrm{Y}$ & NA & $\bar{Y}$ \\
\hline 11 & Fezeu & 2015 & $R$ & 4 & $\mathrm{Y}$ & $\mathrm{Y}$ & $\mathrm{Y}$ & $\mathrm{Y}$ & $\mathrm{Y}$ & $\mathrm{Y}$ & $Y$ & $\mathrm{NA}$ & $\mathrm{Y}$ \\
\hline 12 & Moon & 2014 & $R$ & 4 & $Y$ & $Y$ & $\mathrm{Y}$ & $\mathrm{Y}$ & $Y$ & $\mathrm{Y}$ & $Y$ & $\mathrm{NA}$ & $\bar{Y}$ \\
\hline 1 & Park & 2014 & $R$ & 4 & $\mathrm{Y}$ & $\mathrm{Y}$ & $\mathrm{Y}$ & $\mathrm{Y}$ & $\mathrm{Y}$ & $\mathrm{Y}$ & $\mathrm{Y}$ & $\mathrm{Y}$ & $\mathrm{Y}$ \\
\hline 23 & Bacciu & 2014 & $R$ & 4 & $Y$ & $Y$ & $Y$ & $Y$ & $Y$ & $Y$ & $Y$ & $\mathrm{NA}$ & $\bar{Y}$ \\
\hline 24 & $\mathrm{Li}$ & 2014 & $R$ & 4 & $Y$ & $Y$ & $Y$ & $Y$ & $Y$ & $Y$ & $Y$ & $\mathrm{NA}$ & $\bar{Y}$ \\
\hline 25 & Lee & 2013 & $R$ & 4 & $\mathrm{Y}$ & $Y$ & $\mathrm{Y}$ & $\mathrm{Y}$ & $\mathrm{Y}$ & $\mathrm{Y}$ & $Y$ & $\mathrm{NA}$ & $\mathrm{Y}$ \\
\hline 26 & Bacciu & 2013 & $R$ & 4 & $\mathrm{Y}$ & $\mathrm{Y}$ & $\mathrm{N}$ & $\mathrm{Y}$ & $\mathrm{Y}$ & $\mathrm{Y}$ & $\mathrm{Y}$ & NA & $\bar{Y}$ \\
\hline 27 & $\mathrm{Li}$ & 2012 & $R$ & 4 & $\mathrm{Y}$ & $\mathrm{Y}$ & $Y$ & $Y$ & $Y$ & $Y$ & $Y$ & $\mathrm{NA}$ & $\mathrm{Y}$ \\
\hline 28 & Gross & 2012 & $R$ & 4 & $Y$ & $Y$ & $Y$ & $Y$ & $Y$ & $Y$ & $Y$ & NA & $\mathrm{Y}$ \\
\hline 29 & Mowry & 2012 & $R$ & 4 & $Y$ & $Y$ & $\mathrm{Y}$ & $Y$ & $Y$ & $Y$ & $Y$ & $\mathrm{NA}$ & $Y$ \\
\hline 30 & Lee & 2011 & $R$ & 4 & $Y$ & $Y$ & $Y$ & $Y$ & $Y$ & $Y$ & $Y$ & $\mathrm{NA}$ & $Y$ \\
\hline 31 & Gunther & 2010 & $R$ & 4 & $Y$ & $Y$ & $\mathrm{Y}$ & $Y$ & $Y$ & $Y$ & $\mathrm{Y}$ & $\mathrm{NA}$ & $\mathrm{Y}$ \\
\hline 32 & Back & 2010 & $R$ & 4 & $\mathrm{Y}$ & $Y$ & $\mathrm{Y}$ & $Y$ & $Y$ & $Y$ & $Y$ & $\mathrm{NA}$ & $Y$ \\
\hline 33 & Guzzo & 2009 & $R$ & 4 & $\mathrm{Y}$ & $Y$ & $\mathrm{~N}$ & $\mathrm{Y}$ & $\mathrm{Y}$ & $\mathrm{Y}$ & $\mathrm{Y}$ & $N A$ & $\mathrm{Y}$ \\
\hline 34 & McMonagle & 2008 & $R$ & 4 & $\mathrm{Y}$ & $\mathrm{Y}$ & $\mathrm{Y}$ & $\mathrm{Y}$ & $\mathrm{Y}$ & $\mathrm{Y}$ & $\mathrm{Y}$ & $\mathrm{NA}$ & $\mathrm{Y}$ \\
\hline 35 & Kohmura & 2007 & $R$ & 4 & $\mathrm{Y}$ & $\mathrm{Y}$ & $\mathrm{Y}$ & $\mathrm{Y}$ & $Y$ & $\mathrm{Y}$ & $Y$ & $\mathrm{NA}$ & $Y$ \\
\hline 36 & Lee & 2007 & $R$ & 4 & $\mathrm{Y}$ & $\mathrm{Y}$ & $\mathrm{Y}$ & $\mathrm{Y}$ & $\mathrm{Y}$ & $Y$ & $Y$ & $\mathrm{NA}$ & $\mathrm{Y}$ \\
\hline 37 & Kida & 2007 & $R$ & 4 & $Y$ & $Y$ & $Y$ & $Y$ & $\mathrm{Y}$ & $Y$ & $Y$ & $\mathrm{NA}$ & $\mathrm{Y}$ \\
\hline 38 & Liter & 2007 & $P$ & 4 & $\mathrm{Y}$ & $\mathrm{Y}$ & $\mathrm{Y}$ & $Y$ & $\mathrm{Y}$ & $\mathrm{Y}$ & $Y$ & $\mathrm{NA}$ & $Y$ \\
\hline 6 & Perez & 2005 & $R$ & 4 & $\mathrm{Y}$ & $\mathrm{Y}$ & $\mathrm{Y}$ & $\mathrm{Y}$ & $\mathrm{Y}$ & $\mathrm{Y}$ & $Y$ & $\mathrm{NA}$ & $Y$ \\
\hline 39 & Minovi & 2004 & $R$ & 4 & $\mathrm{Y}$ & $\mathrm{Y}$ & $\mathrm{Y}$ & $\mathrm{Y}$ & $\mathrm{Y}$ & $\mathrm{Y}$ & $\mathrm{Y}$ & NA & $\mathrm{Y}$ \\
\hline 40 & Chung & 2004 & $R$ & 4 & $\mathrm{Y}$ & $\mathrm{Y}$ & $\mathrm{Y}$ & $\mathrm{Y}$ & $\mathrm{Y}$ & $\mathrm{Y}$ & $\mathrm{Y}$ & $\mathrm{NA}$ & $\mathrm{Y}$ \\
\hline 10 & Nadeau & 2003 & $R$ & 4 & $\mathrm{Y}$ & $\mathrm{Y}$ & $\mathrm{Y}$ & $\mathrm{Y}$ & $\mathrm{Y}$ & $\mathrm{Y}$ & $Y$ & $\mathrm{NA}$ & $\mathrm{Y}$ \\
\hline 41 & Kim & 2003 & $R$ & 4 & $Y$ & $Y$ & $Y$ & $Y$ & $Y$ & $\mathrm{Y}$ & $Y$ & $N A$ & $Y$ \\
\hline 42 & Liu & 2001 & $R$ & 4 & $\mathrm{~N}$ & $Y$ & $Y$ & $Y$ & $Y$ & $Y$ & $Y$ & $\mathrm{NA}$ & $Y$ \\
\hline 43 & Chong & 2000 & $R$ & 4 & $Y$ & $Y$ & $Y$ & $Y$ & $Y$ & $Y$ & $Y$ & $\mathrm{NA}$ & $Y$ \\
\hline
\end{tabular}

Abbreviations: N, no; NA, not applicable; NIH, National Institutes of Health; P, prospective; PY, publication year; R, retrospective; Ref., reference number; Y, yes. Note: Numbers are based on questions from - Fig. 1.

did not predict the development of facial weakness. Interestingly, tumor diameter was not a predictor, but the total number of facial nerve segments involved is positively associated with a higher HB grade.

Table 3 Patient demographics

\begin{tabular}{|l|l|}
\hline Age $(n=378)$ & $43.7+/-14.8$ y old \\
\hline Gender $(n=364)$ & $44.8 \%$ male; $55.2 \%$ female \\
\hline Laterality $(n=119)$ & $46.2 \%$ left; $53.8 \%$ right \\
\hline
\end{tabular}

The location of the facial schwannoma had a major impact on the likelihood of facial weakness. Intradural and extratemporal facial schwannomas had a low incidence of facial weakness, whereas intratemporal tumors had a high incidence of facial weakness. - Fig. 4 shows $^{44}$ the likelihood of a higher HB grade by facial nerve segment.

\section{Hearing Loss}

- Table 8 shows the analysis of factors associated with hearing loss. Gender and laterality were not predictors of hearing loss. Older age was correlated with hearing loss. As 


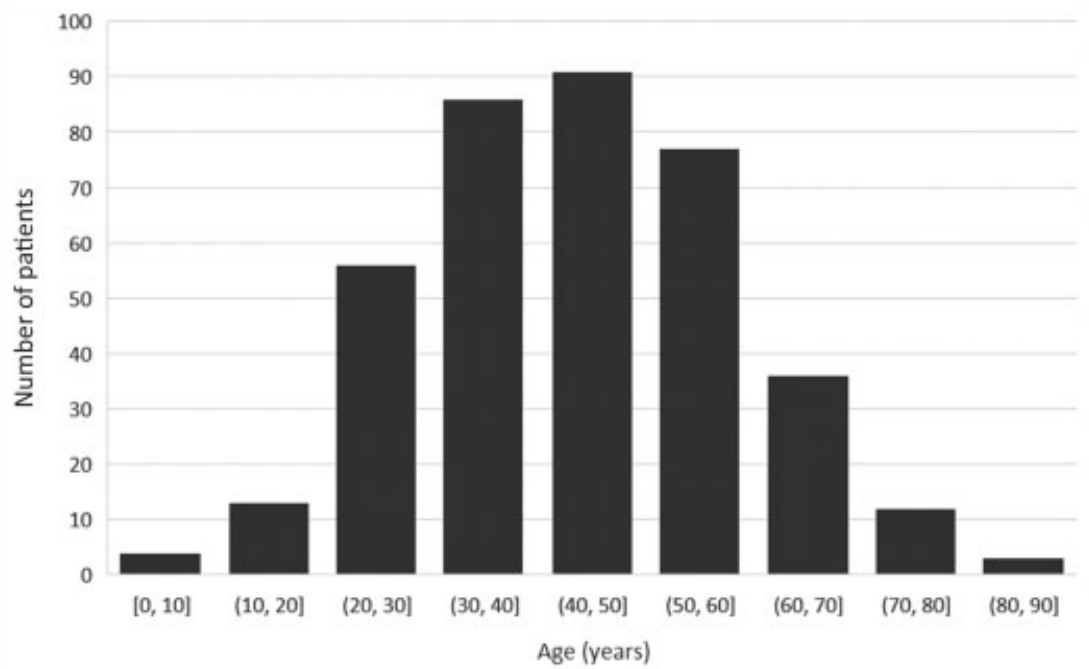

Fig. 3 Patient ages at presentation $(n=378)$.

Table 4 Tumor location by facial nerve segment involvement and sites with all studies included $(n=504)$

\begin{tabular}{|l|l|l|}
\hline Segments involved & $\boldsymbol{n}$ & $\%$ \\
\hline CPA & 119 & $23.6 \%$ \\
\hline IAC & 185 & $36.7 \%$ \\
\hline Labyrinthine & 127 & $25.2 \%$ \\
\hline GG & 198 & $39.3 \%$ \\
\hline Tympanic & 181 & $35.9 \%$ \\
\hline Mastoid & 147 & $29.2 \%$ \\
\hline Parotid & 127 & $25.2 \%$ \\
\hline No. of segments involved & $n$ & $\%$ \\
\hline 1 & 197 & $39.1 \%$ \\
\hline 2 & 161 & $31.9 \%$ \\
\hline 3 & 64 & $12.7 \%$ \\
\hline 4 & 52 & $10.3 \%$ \\
\hline 5 & 18 & $3.6 \%$ \\
\hline 6 & 9 & $1.8 \%$ \\
\hline 7 & 3 & $0.6 \%$ \\
\hline Sites involved & $n$ & $\%$ \\
\hline Intradural & 217 & $43.1 \%$ \\
\hline Intratemporal & 309 & $61.3 \%$ \\
\hline Extratemporal & 127 & $25.2 \%$ \\
\hline No. of sites involved & $n$ & $\%$ \\
\hline 1 & 361 & $71.6 \%$ \\
\hline 2 & 137 & $27.2 \%$ \\
\hline 3 & 6 & $1.2 \%$ \\
\hline
\end{tabular}

Abbreviations: CPA, cerebellopontine angle; GG, geniculate ganglion; IAC, internal auditory canal; No., number.
Table 5 Tumor location by facial nerve segment involvement and sites with studies focusing on specific populations excluded $(n=247)$

\begin{tabular}{|c|c|c|}
\hline Segments involved & $\bar{n}$ & $\%$ \\
\hline CPA & 68 & $27.5 \%$ \\
\hline IAC & 105 & $42.5 \%$ \\
\hline Labyrinthine & 81 & $32.8 \%$ \\
\hline GG & 121 & $49.0 \%$ \\
\hline Tympanic & 114 & $46.2 \%$ \\
\hline Mastoid & 80 & $32.4 \%$ \\
\hline Parotid & 34 & $13.8 \%$ \\
\hline No. of segments involved & $n$ & $\%$ \\
\hline 1 & 80 & $32.4 \%$ \\
\hline 2 & 69 & $27.9 \%$ \\
\hline 3 & 40 & $16.2 \%$ \\
\hline 4 & 37 & $15.0 \%$ \\
\hline 5 & 11 & $4.5 \%$ \\
\hline 6 & 8 & $3.2 \%$ \\
\hline 7 & 2 & $0.8 \%$ \\
\hline Sites involved & $n$ & $\%$ \\
\hline Intradural & 123 & $49.8 \%$ \\
\hline Intratemporal & 177 & $71.7 \%$ \\
\hline Extratemporal & 34 & $13.8 \%$ \\
\hline No. of sites involved & $n$ & $\%$ \\
\hline 1 & 165 & $66.8 \%$ \\
\hline 2 & 77 & $31.2 \%$ \\
\hline 3 & 5 & $2.0 \%$ \\
\hline
\end{tabular}

Abbreviations: CPA, cerebellopontine angle; GG, geniculate ganglion; IAC, internal auditory canal; No., number. 
Table 6 Frequencies of presenting symptoms $(n=401)$.

\begin{tabular}{|l|l|}
\hline Presenting symptom & Frequency \\
\hline Facial palsy & $51.2 \%$ \\
\hline HB 1 & $48.8 \%$ \\
\hline HB 2 & $15.1 \%$ \\
\hline HB 3 & $15.3 \%$ \\
\hline HB 4 & $5.8 \%$ \\
\hline HB 5 & $7.3 \%$ \\
\hline HB 6 & $7.7 \%$ \\
\hline HL & $43.1 \%$ \\
\hline Sensorineural HL & $53.3 \%$ \\
\hline Conductive HL & $42.7 \%$ \\
\hline Mixed HL & $4.0 \%$ \\
\hline Tinnitus & $21.7 \%$ \\
\hline Vertigo/imbalance & $20.2 \%$ \\
\hline Parotid mass & $18.7 \%$ \\
\hline Facial spasm & $5.2 \%$ \\
\hline Facial pain & $3.2 \%$ \\
\hline Otalgia & $3.0 \%$ \\
\hline Aural fullness & $1.7 \%$ \\
\hline Otoscopic finding & $1.2 \%$ \\
\hline Dysgeusia & $1.0 \%$ \\
\hline Hypesthesia & $1.0 \%$ \\
\hline Otorrhea & $0.5 \%$ \\
\hline Headaches & $0.5 \%$ \\
\hline Incidental finding & $0.5 \%$ \\
\hline Hyperlacrimation & $0.5 \%$ \\
\hline Xerophthalmia & $0.3 \%$ \\
\hline
\end{tabular}

Abbreviations: $\mathrm{HB}$, House-Brackmann grade; $\mathrm{HL}$, hearing loss.

with facial weakness, tumor diameter was not a predictor but the number of facial nerve segments involved was a positive predictor for hearing loss.

Location was also important for predicting the likelihood of hearing loss. The more proximal the involvement of the facial nerve, the more likely there was to be hearing loss. - Fig. 5 shows ${ }^{44}$ the OR for each facial nerve segment.

Among patients who had sensorineural and conductive hearing loss documented $(n=75), 53.3 \%$ were sensorineural, $42.7 \%$ were conductive, and $4.0 \%$ had a mixed loss. Each patient with documented sensorineural hearing loss had intradural involvement and every patient with conductive hearing loss had intratemporal involvement.

\section{Discussion}

Treatment for facial schwannomas is a delicate balancing act, so understanding the characteristics that are more closely correlated with morbidity will help the surgeon and patient decide the best course of treatment. Using a unique systema-
Table 7 Predictors of a higher House-Brackmann grade

\begin{tabular}{|l|l|l|l|}
\hline & $n$ & $\begin{array}{l}\text { Odds Ratio } \\
(95 \% \mathrm{Cl})\end{array}$ & $p$ \\
\hline Demographics & & & \\
\hline $\begin{array}{l}\text { Sex } \\
\text { (female vs. male) }\end{array}$ & 364 & $1.13(0.73-1.75)$ & 0.59 \\
\hline Age (y, older) & 378 & $0.99(0.97-1.00)$ & 0.05 \\
\hline $\begin{array}{l}\text { Laterality } \\
\text { (left vs. right) }\end{array}$ & 119 & $2.31(0.99-5.37)$ & 0.05 \\
\hline $\begin{array}{l}\text { Hearing (abnormal } \\
\text { vs. normal) }\end{array}$ & 254 & $0.97(0.55-1.72)$ & 0.92 \\
\hline Tumor extent & 93 & $0.95(0.89-1.01)$ & 0.10 \\
\hline $\begin{array}{l}\text { Tumor diameter } \\
\text { (mm) }\end{array}$ & 504 & $1.40(1.20-1.63)$ & $<0.001$ \\
\hline $\begin{array}{l}\text { Facial nerve } \\
\text { segments } \\
\text { involved }\end{array}$ & 504 & $4.78(2.66-8.58)$ & $<0.001$ \\
\hline Sites & 504 & $0.68(0.34-1.34)$ & 0.27 \\
\hline Intradural & 504 & $0.56(0.37-0.83)$ & 0.004 \\
\hline Intratemporal & & & \\
\hline Extratemporal & & & \\
\hline
\end{tabular}

Abbreviation: $\mathrm{Cl}$, confidence interval.

${ }^{a}$ House-Brackmann grade collapsed into 1-2 and 3-6 because proportional odds assumption violated.

tic review study design that only includes studies with detailed patient data, we were able to obtain a sample size unprecedented in the literature. This level of detail allowed meaningful conclusions relevant to the management of facial schwannomas. First, it provides epidemiologic data with regard to demographics, tumor location, and clinical presentation. Second, we found that the number of facial nerve segments involved by tumor is a better predictor than tumor diameter for both facial weakness and hearing loss. Third, we learned intratemporal tumor location is a predictor for facial weakness. Finally, the more proximal the schwannoma is located, the more likely there is to be hearing loss. The term predictor in this study refers to variables associated with morbidity at presentation, this is not examining future prognosis with observation.

The epidemiologic data accumulated in this study offers some value to the literature given the multi-institutional nature of a systematic review and the large sample size. The average age was 43.7 years old, with a slight preponderance toward females and right sided neoplasms. There is wide variability in the age at presentation and the distribution across that span follows a normal distribution. The largest clinical studies that have been performed ${ }^{7,34,45}$ which largely were not used in this study because the data was understandably aggregated, also had average ages in the fifth decade of life.

We found that the most common facial nerve segment involved was the geniculate ganglion which was closely followed by the tympanic segment and the IAC. When 


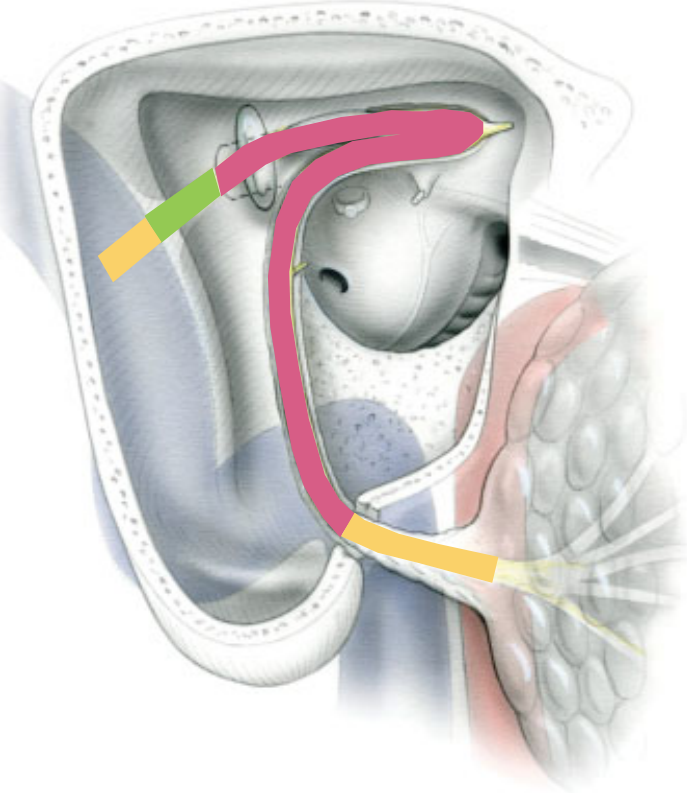

\begin{tabular}{|l|c|c|}
\hline Location & Odds Ratio $(95 \%$ Cl) & $p$ \\
\hline CPA $^{*}$ & $0.64(0.37-1.09)$ & 0.10 \\
\hline IAC & $0.59(0.40-0.88)$ & 0.01 \\
\hline Labyrinthine & $1.57(1.03-2.40)$ & 0.04 \\
\hline GG $^{*}$ & $2.75(1.70-4.44)$ & $<0.001$ \\
\hline Tympanic & $3.56(2.39-5.30)$ & $<0.001$ \\
\hline Mastoid & $1.84(1.24-2.73)$ & 0.002 \\
\hline Parotid* & $0.68(0.34-1.34)$ & 0.27 \\
\hline
\end{tabular}

Fig. 4 Predictors of higher House-Brackmann grade by facial nerve segment involvement. *House-Brackmann grade collapsed into 1-2 and 36 because proportional odds assumption violated. Cl, confidence interval; CPA, cerebellopontine angle; IAC, internal auditory canal; GG, geniculate ganglion. Source ${ }^{44}$

studies that focused on specific populations were excluded, the frequency of extratemporal schwannomas decreased and the other locations increased proportionally. We believe the latter totals are likely a more accurate representation of incidence of involvement of the various facial nerve segments by facial schwannomas. The geniculate ganglion, IAC, and tympanic segments are consistently the three most common sites of involvement in the largest studies performed previously. ${ }^{7,34,45}$ The greater superficial petrosal

Table 8 Predictors of hearing loss

\begin{tabular}{|l|l|l|l|}
\hline & $n$ & $\begin{array}{l}\text { Odds Ratio } \\
(95 \% \mathrm{Cl})\end{array}$ & $p$ \\
\hline Demographics & & & \\
\hline $\begin{array}{l}\text { Sex } \\
\text { (female vs. male) }\end{array}$ & 140 & $1.35(0.67-2.75)$ & 0.40 \\
\hline Age (y, older) & 140 & $1.04(1.01-1.07)$ & 0.008 \\
\hline $\begin{array}{l}\text { Side } \\
\text { (left vs. right) }\end{array}$ & 71 & $0.99(0.34-2.86)$ & 0.99 \\
\hline Tumor extent & & & 0.19 \\
\hline $\begin{array}{l}\text { Tumor diameter } \\
\text { (mm) }\end{array}$ & 60 & $0.97(0.92-1.02)$ & 0.003 \\
\hline $\begin{array}{l}\text { Facial nerve } \\
\text { segments } \\
\text { Involved }\end{array}$ & 254 & $1.43(1.13-1.82)$ & \\
\hline Sites & 254 & $3.26(1.88-5.65)$ & $<0.001$ \\
\hline Intradural & 254 & $0.60(0.30-1.19)$ & 0.14 \\
\hline Intratemporal & 254 & $0.27(0.09-0.77)$ & 0.01 \\
\hline Extratemporal & & \\
\hline
\end{tabular}

Abbreviation: $\mathrm{Cl}$, confidence interval. nerve, ${ }^{46}$ nerve to stapedius, ${ }^{47}$ and chorda tympani nerve ${ }^{48}$ have all been reported involved in case reports; however, they are extraordinarily rare. In studies used for this systematic review, there were only two patients with greater superficial petrosal branch involvement and both were in large tumors involving at least five facial nerve segments. ${ }^{34}$ There were no patients with nerve to stapedius or chorda tympani involvement.

Most patients had their presenting symptom(s) reported (79.6\%). The most common presenting symptoms were facial weakness and hearing loss, with tinnitus, vertigo/imbalance, and parotid masses being the next most common. Facial spasm is a relatively uncommon (5.2\%) but notable symptom which can help to differentiate facial schwannomas from a vestibular schwannoma.

It is critical to differentiate a vestibular schwannoma from an intradural facial schwannoma because the treatment paradigm of each is very different. Unfortunately, facial schwannomas confined to the IAC and CPA present similarly to vestibular schwannomas and can be impossible to differentiate preoperatively. A high index of suspicion is warranted if there are any facial nerve signs or symptoms or any extension past the fundus of the IAC radiologically. ${ }^{23}$ Intraoperatively, facial schwannomas can be distinguished by the presence of spontaneous action potentials while drilling the bony IAC, action potentials when stimulating the tumor capsule, or the intimate involvement of the facial nerve with the tumor. ${ }^{49}$ If encountered unexpectedly intraoperative, decompression or subtotal resection should generally be the treatment of choice. ${ }^{23}$

The most valuable conclusions that can be taken from this study are the factors associated with facial nerve weakness and hearing loss. These conclusions cannot predict prognosis 


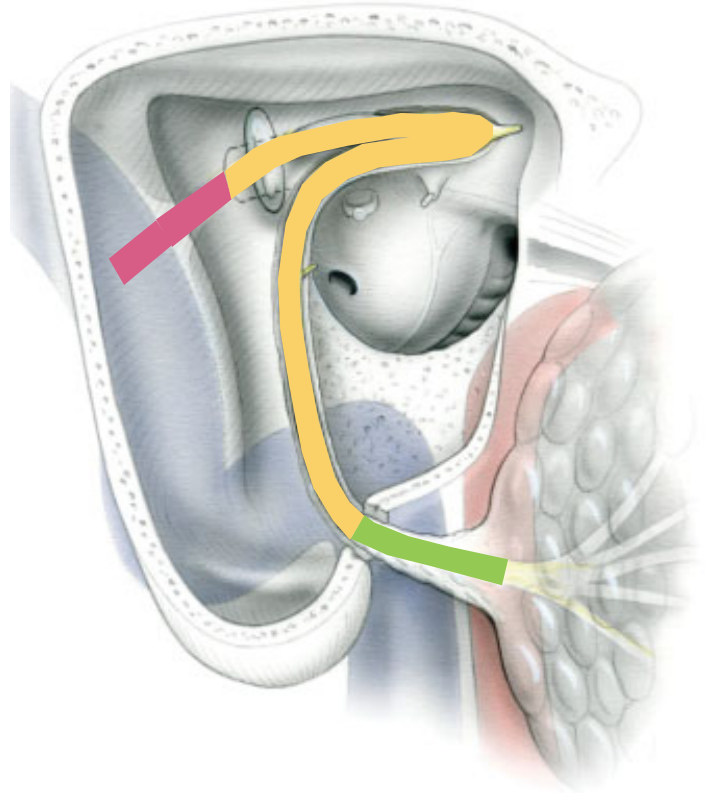

\begin{tabular}{|l|l|c|}
\hline Location & Odds Ratio $(95 \% \mathrm{CI})$ & $\boldsymbol{p}$ \\
\hline CPA & $4.49(2.08-9.70)$ & $<0.001$ \\
\hline IAC & $2.82(1.63-4.86)$ & $<0.001$ \\
\hline Labyrinthine & $1.76(0.94-3.27)$ & 0.08 \\
\hline GG & $0.93(0.54-1.60)$ & 0.79 \\
\hline Tympanic & $1.23(0.71-2.13)$ & 0.45 \\
\hline Mastoid & $0.94(0.54-1.63)$ & 0.82 \\
\hline Parotid & $0.027(0.09-0.77)$ & 0.01 \\
\hline
\end{tabular}

Fig. 5 Predictors of hearing loss by facial nerve segment involvement. $\mathrm{Cl}$, confidence interval; $\mathrm{CPA}$, cerebellopontine angle; IAC, internal auditory canal; GG, geniculate ganglion. Source ${ }^{44}$

because they are based on observations of single time points in the natural course of facial schwannomas. As with vestibular schwannomas, ${ }^{50}$ there is no known way to predict if a facial schwannoma will continue to grow or not. However, it is reasonable to conjecture that if, for example, a patient presents with normal facial function but has variables associated with poor facial nerve function, that further tumor growth is perhaps more likely to cause facial weakness than a patient with variables associated with good facial nerve function. When making decisions regarding timing and type of intervention, knowing these associations could potentially impact clinical decision making.

A greater degree of facial weakness is positively associated with intratemporal tumor locations and negatively associated with intradural and extratemporal tumor locations. The mechanism of facial nerve weakness for facial schwannomas is currently unknown ${ }^{7}$ but based on these findings it is likely related to compression of the nerve and/or vasa nervorum from growth of the neoplasm within the limitations of the fallopian canal. However, it is surprising that the tympanic segment involvement is trending toward being a stronger predictor for facial weakness compared with the labyrinthine segment. Considering the smaller caliber of the labyrinthine segment of the fallopian canal, ${ }^{51}$ one would expect the labyrinthine segment to be more strongly associated with facial weakness.

We also found that the number of facial nerve segments involved is a positive predictor of facial weakness, whereas tumor diameter is not a predictor. This also suggests that facial weakness is likely propagated through a mechanism related to compression. Cell growth will either lead to increased pressure within a confined space or increased tumor volume, so tumors putting more pressure on their affiliated nerve segment will theoretically have less volume than they otherwise would. The fact that many segments are tightly grouped together in the intratemporal segment of the facial nerve could also have influenced this analysis. Age, gender, and laterality did not impact facial nerve status, although older age and left sided tumors were trending toward significance.

Hearing loss was not an inclusion criterion in this study but it was documented in over half of the patients. Unfortunately, across studies the documentation of hearing loss was inconsistent, so our analysis was limited to a simple positive or negative binary metric. The analysis still revealed meaningful results.

A patient was more likely to have hearing loss the more proximal the involvement of their schwannoma along the facial nerve. Intradural involvement, particularly in the CPA, was a positive predictor of hearing loss. Intratemporal involvement was a neutral predictor of hearing loss compared with other locations, and extratemporal involvement was a negative predictor. The mechanism for hearing loss in the IAC and CPA has been studied more extensively in vestibular schwannomas and the exact mechanism is still unclear but it is thought to be from nerve compression with resultant thinning of cochlear nerve fibers and/or impairment of blood supply to the auditory nerve or cochlea. ${ }^{52}$ Hearing loss in patients with intradural facial schwannomas is likely via the same mechanism given the similar anatomic relationships. Intratemporal hearing loss is more likely from a conductive hearing loss secondary to either mass effect within the middle ear, ossicular erosion, or mass effect within the external auditory canal. ${ }^{34}$ Among the relatively small number of patients $(n=75)$ where sensorineural and conductive hearing loss were differentiated, there was a fairly even mix that closely correlated with tumor location in the expected pattern. 
As with facial weakness, the number of facial nerve segments involved was a positively associated with hearing loss whereas tumor diameter was not a predictor. Older age was a positive predictor of hearing loss which is likely because many older patients have hearing loss secondary to nontumor causes. Gender and laterality did not impact the likelihood of hearing loss.

The strengths of this study include the large sample size, the diversity of institutions where the patients presented, the uniformity of the HB grading system used with all patients and the level of detail mandated by the inclusion criteria created. Limitations include the lack of detail in hearing loss data available, the reliance on the reporting of others and the risk of publication bias inherent in a systematic review.

\section{Conclusion}

Facial schwannomas are extremely rare tumors with a wide variety of clinical presentations. The number of facial nerve segments involved were positively associated with both facial weakness and hearing loss, whereas tumor diameter is not a predictor for either. Intratemporal neoplasms are a positively associated with a greater degree of facial weakness. The more proximal a facial schwannoma is along the course of the facial nerve, the more likely a patient is to exhibit hearing loss. The type and timing of intervention should be tailored to individual patients with these findings in mind.

Note

This study was presented as a podium presentation at the North American Skull Base Society $28^{\text {th }}$ Annual Meeting on February 17, 2018 in San Diego, California, U.S.A.

\section{Acknowledgments}

Special thanks to Jeanne Sadlik, MLS from the Loyola University Chicago Health Sciences Library for performing the literature search.

\section{References}

1 Park SH, Kim J, Moon IS, Lee WS. The best candidates for nervesparing stripping surgery for facial nerve schwannoma. Laryngoscope 2014;124(11):2610-2615

2 McRackan TR, Wilkinson EP, Rivas A. Primary tumors of the facial nerve. Otolaryngol Clin North Am 2015;48(03):491-500

3 Shi W, Jain V, Kim H, et al. Fractionated stereotactic radiotherapy for facial nerve schwannomas. J Neurol Surg B Skull Base 2016;77 (01):75-80

4 McRackan TR, Rivas A, Wanna GB, et al. Facial nerve outcomes in facial nerve schwannomas. Otol Neurotol 2012;33(01):78-82

5 Yang W, Zhao J, Han Y, et al. Long-term outcomes of facial nerve schwannomas with favorable facial nerve function: tumor growth rate is correlated with initial tumor size. Am J Otolaryngol 2015; 36(02):163-165

6 Perez R, Chen JM, Nedzelski JM. Intratemporal facial nerve schwannoma: a management dilemma. Otol Neurotol 2005;26 (01):121-126

7 Carlson ML, Deep NL, Patel NS, et al. Facial nerve schwannomas: Review of 80 cases over 25 years at Mayo Clinic. Mayo Clin Proc 2016;91(11):1563-1576
8 Sun Y, Xing B, Han S, et al. Stripping surgery in facial nerve schwannomas with favorable facial nerve function. Am J Otolaryngol 2015;36(04):513-516

9 Lu R, Li S, Zhang L, Li Y, Sun Q. Stripping surgery in intratemporal facial nerve schwannomas with poor facial nerve function. Am J Otolaryngol 2015;36(03):338-341

10 Nadeau DP, Sataloff RT. Fascicle preservation surgery for facial nerve neuromas involving the posterior cranial fossa. Otol Neurotol 2003;24(02):317-325

11 Fezeu F, Lee CC, Dodson BK, et al. Stereotactic radiosurgery for facial nerve schwannomas: a preliminary assessment and review of the literature. Br J Neurosurg 2015;29(02):213-218

12 Moon JH, Chang WS, Jung HH, Lee KS, Park YG, Chang JH. Gamma knife surgery for facial nerve schwannomas. J Neurosurg 2014; 121(Suppl):116-122

13 Madhok R, Kondziolka D, Flickinger JC, Lunsford LD. Gamma knife radiosurgery for facial schwannomas. Neurosurgery 2009;64 (06):1102-1105, discussion 1105

14 Moher D, Liberati A, Tetzlaff J, Altman DG; PRISMA Group. Preferred reporting items for systematic reviews and meta-analyses: the PRISMA statement. J Clin Epidemiol 2009;62(10): 1006-1012

15 House JW, Brackmann DE. Facial nerve grading system. Otolaryngol Head Neck Surg 1985;93(02):146-147

16 Centre for evidence-based medicine. Available from: https:// www.cebm.net/; accessed on June 8, 2017

17 American Academy of Otolaryngology-Head and Neck Surgery Foundation, Inc. Committee on Hearing and Equilibrium guidelines for the evaluation of hearing preservation in acoustic neuroma (vestibular schwannoma). American Academy of Otolaryngology-Head and Neck Surgery Foundation, INC. Otolaryngol Head Neck Surg 1995;113(03):179-180

18 Harris PA, Taylor R, Thielke R, Payne J, Gonzalez N, Conde JG. Research electronic data capture (REDCap)-a metadata-driven methodology and workflow process for providing translational research informatics support. J Biomed Inform 2009;42(02):377-381

19 Study quality assessment tools: quality assessment tool for case series studies. National Institutes of Health. https://www.nhlbi. nih.gov/health-pro/guidelines/in-develop/cardiovascular-riskreduction/tools/case_series. Accessed on June 8, 2017

20 Zheng Z, Li J, Shen Y, Xu L, Sun J. Radical intracapsular microenucleation technique for exclusively intraparotid facial nerve schwannoma: Long-term follow-up review. J Craniomaxillofac Surg 2016;44(12):1963-1969

21 Xiang D, Liu L, Li Y, Qiao J. Near-total removal of facial nerve schwannomas: long-term outcomes. Am J Otolaryngol 2015;36 (03):390-392

22 Doshi J, Heyes R, Freeman SR, et al. Clinical and radiological guidance in managing facial nerve schwannomas. Otol Neurotol 2015;36(05):892-895

23 Bacciu A, Medina M, Ben Ammar M, et al. Intraoperatively diagnosed cerebellopontine angle facial nerve schwannoma: how to deal with it. Ann Otol Rhinol Laryngol 2014;123(09):647-653

24 Li Y, Liu H, Cheng Y. Subtotal resection of facial nerve schwannoma is not safe in the long run. Acta Otolaryngol 2014;134(04): 433-436

25 Lee DW, Byeon HK, Chung HP, Choi EC, Kim S-H, Park YM. Diagnosis and surgical outcomes of intraparotid facial nerve schwannoma showing normal facial nerve function. Int J Oral Max. 2013;42(07):874-879

26 Bacciu A, Nusier A, Lauda L, Falcioni M, Russo A, Sanna M. Are the current treatment strategies for facial nerve schwannoma appropriate also for complex cases? Audiol Neurootol 2013;18(03):184-191

$27 \mathrm{Li} Y$, Jiang $\mathrm{H}$, Chen X, et al. Management options for intraparotid facial nerve schwannoma. Acta Otolaryngol 2012;132(11):1232-1238

28 Gross BC, Carlson ML, Moore EJ, Driscoll CL, Olsen KD. The intraparotid facial nerve schwannoma: a diagnostic and management conundrum. Am J Otolaryngol 2012;33(05):497-504 
29 Mowry S, Hansen M, Gantz B. Surgical management of internal auditory canal and cerebellopontine angle facial nerve schwannoma. Otol Neurotol 2012;33(06):1071-1076

30 Lee WS, Kim J. Revised surgical strategy to preserve facial function after resection of facial nerve schwannoma. Otol Neurotol 2011; 32(09):1548-1553

31 Günther M, Danckwardt-Lillieström N, Gudjonsson O, et al. Surgical treatment of patients with facial neuromas-a report of 26 consecutive operations. Otol Neurotol 2010;31(09):1493-1497

32 Bäck L, Heikkilä T, Passador-Santos F, Saat R, Leivo I, Mäkitie AA. Management of facial nerve schwannoma: A single institution experience. Acta Otolaryngol 2010;130(10):1193-1198

33 Guzzo M, Ferraro L, Ibba T, et al. Schwannoma in the parotid gland. Experience at our institute and review of the literature. Tumori 2009;95(06):846-851

34 McMonagle B, Al-Sanosi A, Croxson G, Fagan P. Facial schwannoma: results of a large case series and review. J Laryngol Otol 2008;122(11):1139-1150

35 Kohmura E, Aihara H, Miyake S, Fujita A. Intradural facial nerve schwannoma: diagnostic and therapeutic problems. Skull Base 2007;17(03):215-222

36 Lee JD, Kim SH, Song MH, Lee HK, Lee WS. Management of facial nerve schwannoma in patients with favorable facial function. Laryngoscope 2007;117(06):1063-1068

37 Kida Y, Yoshimoto M, Hasegawa T. Radiosurgery for facial schwannoma. J Neurosurg 2007;106(01):24-29

38 Litre CF, Gourg GP, Tamura M, et al. Gamma knife surgery for facial nerve schwannomas. Neurosurgery 2007;60(05):853-859, discussion 853-859

39 Minovi A, Vosschulte R, Hofmann E, Draf W, Bockmühl U. Facial nerve neuroma: surgical concept and functional results. Skull Base 2004;14(04):195-200, discussion 200-201

40 Chung JW, Ahn JH, Kim JH, Nam SY, Kim CJ, Lee KS. Facial nerve schwannomas: different manifestations and outcomes. Surg Neurol 2004;62(03):245-252, discussion 452
41 Kim CS, Chang SO, Oh SH, Ahn SH, Hwang CH, Lee HJ. Management of intratemporal facial nerve schwannoma. Otol Neurotol 2003; 24(02):312-316

42 Liu R, Fagan P. Facial nerve schwannoma: surgical excision versus conservative management. Ann Otol Rhinol Laryngol 2001;110 (11):1025-1029

43 Chong KW, Chung YFA, Khoo MLC, Lim DTH, Hong GS, Soo KC. Management of intraparotid facial nerve schwannomas. Aust NZ J Surg 2000;70(10):732-734

44 Jackler RK. Atlas of Skull Base Surgery and Neurotology. 2nd ed. Stuttgart, Germany; Thieme: 2008

45 Wilkinson EP, Hoa M, Slattery WH III, et al. Evolution in the management of facial nerve schwannoma. Laryngoscope 2011; 121(10):2065-2074

46 Umredkar A, Singla N, Gupta SK, Radotra B. Greater superficial petrosal nerve schwannoma: report of two cases and literature review. Neurol India 2011;59(05):786-788

47 Lipkin AF, Coker NJ, Jenkins HA, Alford BR. Intracranial and intratemporal facial neuroma. Otolaryngol Head Neck Surg 1987;96(01):71-79

48 Huoh KC, Cheung SW. Chorda tympani neuroma. Otol Neurotol 2010;31(07):1172-1173

49 McNulty BN, Wise S, Cohen DS, et al. Facial nerve schwannomas mimicking as vestibular schwannomas. J Neurol Surg B Skull Base 2017;78(04):283-287

50 Jethanamest D, Rivera AM, Ji H, Chokkalingam V, Telischi FF, Angeli SI. Conservative management of vestibular schwannoma: Predictors of growth and hearing. Laryngoscope 2015;125(09):2163-2168

51 Vianna M, Adams M, Schachern P, Lazarini PR, Paparella MM, Cureoglu S. Differences in the diameter of facial nerve and facial canal in bell's palsy-a 3-dimensional temporal bone study. Otol Neurotol 2014;35(03):514-518

52 Sughrue ME, Kane AJ, Kaur R, et al. A prospective study of hearing preservation in untreated vestibular schwannomas. J Neurosurg 2011;114(02):381-385

\section{Appendix A}

Search strategies

1. PubMed-NCBI: Facial nerve (MeSH) and Neuroma (MeSH: No Exp) or ("facial nerve" or "seventh cranial nerve") and neuroma or "facial neuroma" or "facial nerve schwannoma." English only filter used.

2. Scopus: Keyword search-“Facial nerve neuroma” or "seventh cranial nerve neuroma" or "Facial neuroma" or "Facial nerve schwannoma" or "Facial schwannoma." English only filter used. 